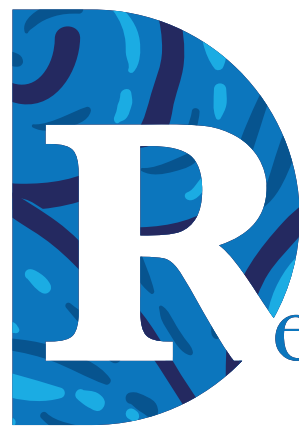

\title{
EALIDAD eflexión
}

Reality and Reflection

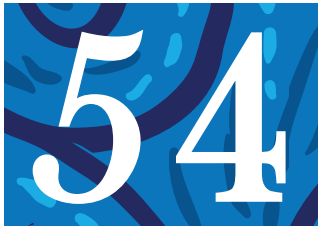

Año 21, N 54, Extraordinario, San Salvador, El Salvador, Centroamérica. Revista Semestral 2021

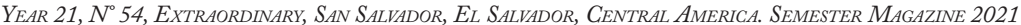

\section{El caracol tintóreo (Plicopurpura pansa) en los municipios de Jicalapa y Chiltiupán, La Libertad, E1 Salvador ${ }^{1}$}

The dye snail (Plicopurpura pansa) in the municipalities of Jicalapa and Chiltiupán, La Libertad, El Salvador

Ana María Rivera Licenciatura en Biología de la Universidad de El Salvador Curadora del Museo de Historia Natural de El Salvador, Ministerio de Cultura, El Salvador arivera@cultura.gob.sv 


\section{RESUMEN}

El estudio fue realizado en dos áreas rocosas del país: las playas Shutia y El Balsamar, pertenecientes al departamento de La Libertad. Los objetivos de este estudio fueron conocer las técnicas de sexado y la proporción de machos y hembras en ambos puntos de muestreo; el método de captura y recaptura estuvo orientado a investigar la densidad poblacional del caracol púrpura, y también conocer parte de la biología del caracol Plicopurpura pansa.

Palabras clave: caracol tintóreo, densidad, largo, biología, El Salvador.

\section{ABSTRACT}

The study was conducted in two rocky areas of the country: Shutia beach and El Balsamar beach, both belonging to the department of La Libertad. The objectives of the study were to know the sexing technique and the proportion of males and females in both sampling points; the capture and recapture method was applied to investigate the population density of the purple snail, and also to know part of the biology of the Plicopurpura pansa snail.

Keyzords: dye purple snail, density, length, biology, El Salvador. 


\section{Introducción}

Plicopurpura pansa es una especie de caracol de mar, un molusco gasterópodo marino de la familia Muricidae, o caracoles de roca. Su distribución geográfica abarca desde Baja California Sur hasta las islas Galápagos y Norte de Perú (Keen, 1971), considerándose como una especie típica de la provincia panámica, que vive en la franja mesolitoral del litoral. El factor limitante para su distribución son las bajas temperaturas (Acevedo y Hernández, 1988; Villalpando, 1986; León, 1989; citados en Garza et al., 2007).

El conocimiento de la extracción del tinte por las culturas prehispánicas de la costa pacífica, se tiene desde hace más de un milenio. El tinte es utilizado para teñir hilos de algodón, prendas de vestir, ceremoniales y mortuoria como lo atestiguan textiles encontrados en Paracas (Perú), y en la Cueva Chiptic en Chiapas, México (Nutall, 1909, citado en Payne, 2017); siendo un recurso cultural económicamente importante para etnias de Oaxaca (México) y Borucas en Costa Rica (Quintanilla, 2012), las cuales han estado relacionadas con el poder o la fertilidad y la cultura mágico-religiosa de los grupos indígenas que se han dedicado a esta actividad (Turok et al., 1988).

Según registros históricos esto condujo a una sobreexplotación de $P$. pansa entre 1529 y 1821 en América Central, y tuvo un impacto drástico en la disminución de la población (Ramírez-Rodríguez y Naegel, 2003). El impacto de la conquista y colonización tuvo efectos directos sobre un sin número de formas de vida y prácticas legendarias de las sociedades nativas, en especial en las prácticas de extracción del caracol de tinte en las sociedades indígenas. Según Payne (2017), en años recientes hubo similar situación en el estado de Oaxaca, México (Turok et al., 1988); por lo que el gobierno de México prohibió la explotación comercial de P.pansa, exceptuando a las poblaciones indígenas.

Ante esta situación, se han generado diversos estudios en la costa pacífica mexicana para conocer el estado de la población, hábitos alimenticios y aspectos reproductivos de la especie. No obstante, en E1 Salvador ha sido nulo el conocimiento de la biología y estado poblacional de P. pansa.

En base a lo expuesto se planteó determinar la densidad poblacional y la proporción de hembrasmachos en zona rocosa del departamento de La Libertad, El Salvador, para conocer la densidad poblacional de Plicopurpura.

\section{Método}

El estudio se realizó en dos playas rocosas de la costa de El Salvador; playa Shutia, Chiltiupán $\left(13.498938^{\circ} \mathrm{N}-89.466348^{\circ} \mathrm{O}\right)$ y playa El Balsamar, Jicalapa $\left(13.499461^{\circ} \mathrm{N}-89.539547^{\circ} \mathrm{O}\right)$. El trabajo se ejecutó en los meses de noviembre-diciembre de 2014 y enero-febrero de 2015 . Se realizaron cuatro muestreos en marea negativa de luna nueva (mareas de tiempo prolongado); los muestreos fueron uno 
por mes, definiendo un transepto de $100 \mathrm{~m}$ de largo en cada punto de muestreo colocado paralelo a la línea costera (fotografía 1), y estos fueron georreferenciados.

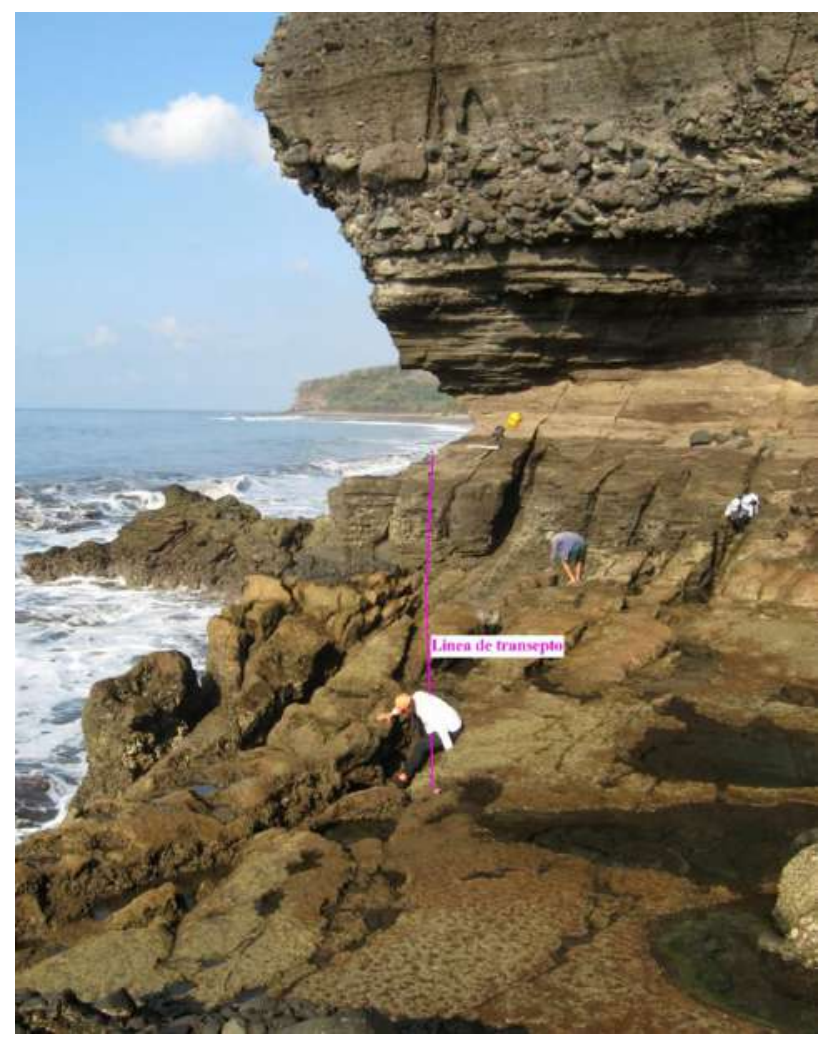

Fotografía 1. Transepto paralelamente a línea costera (playa Shutia). Fuente: recurso propio.

Los ejemplares se desprendieron de las rocas manualmente y de forma cuidadosa para evitar algún daño al animal. Fueron colocados en depósitos plásticos con agua de mar o en pequeñas pozas formadas entre las rocas, para tomar sus datos mirísticos y determinar el sexo por observación directa de la presencia o ausencia de pene.

La longitud de concha se midió con un vernier desde el ápice hasta el canal sifonal de cada ejemplar capturado, con el propósito de conocer la diferencia de tamaño de concha entre hembras y machos.

Los muestreos se realizaron aplicando el método de captura y recaptura, para obtener una estimación más confiable del tamaño de la población en cada sitio trabajado; este método se aplica a poblaciones de organismos móviles (Petersen, 1896, citado en Barbero, 2018; Michel- 
Morfin et al., 2009). El método consiste en marcar un grupo de individuos una vez y liberarlos; la segunda muestra debe ser tomada al azar, es decir, los individuos marcados y no marcados tienen la misma probabilidad de la captura y cada muestra se identifica con un color. En el estudio se trabajó pintura acrílica especial para que no se perdiera con el oleaje y fue aplicada con un pincel en el ápice de cada caracol.

\section{Resultados}

En los cuatro muestreos realizados se obtuvo un total de 138 ejemplares en playa Shutia y 101 en playa E1 Balsamar, obteniendo un promedio de población total para playa Shutia de 75.28 hembras y 94.18 machos; y en playa E1 Balsamar el promedio fue de 60.52 hembras y 65.57 machos. Los datos reflejan que la población muestreada es mayor en Shutia que en E1 Balsamar, y la presencia de machos fue mayor que las hembras, siendo esta diferencia más acentuada en E1 Balsamar (gráfico 1).

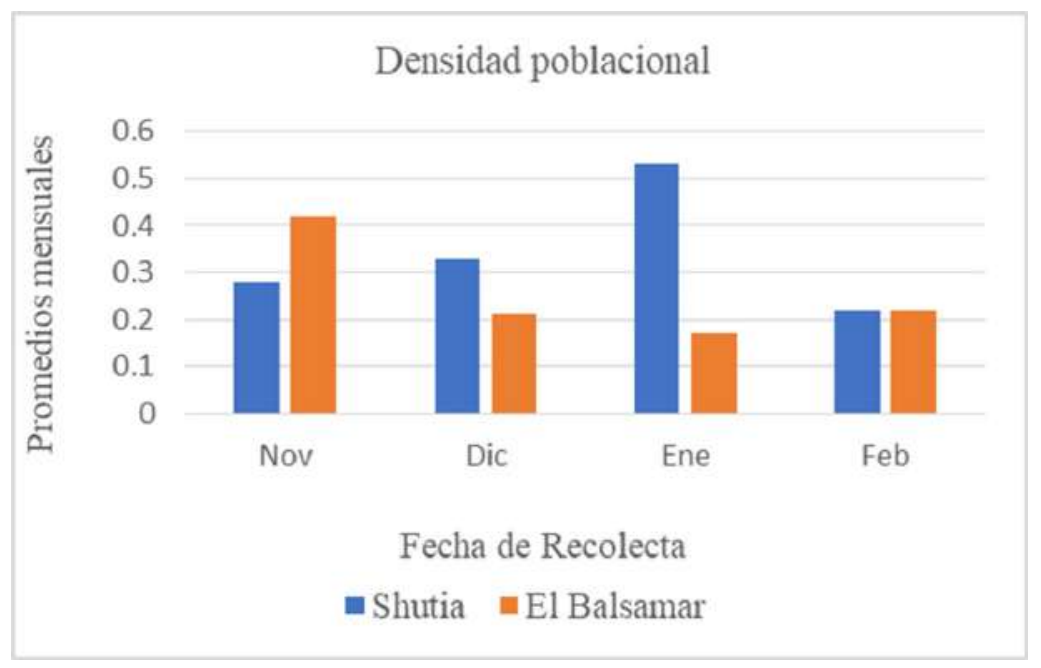

Gráfico 1. Promedios de densidad relativa de ambos puntos muestreados. Fuente: elaboración propia.

La densidad relativa para playa Shutia fue de 1.36 caracoles $/ \mathrm{m}^{2}$ y en playa El Balsamar $1.02 \mathrm{~m}^{2}$, y su proporción sexual Shutia con 0.84 hembras/macho y el Balsamar 0.67 hembras/macho.

En cuanto a longitud de la concha, los caracoles hembras son de mayor tamaño que los machos, siendo el promedio de longitud para hembras $34.85 \mathrm{~mm}$ en Shutia y para Balsamar un $29.81 \mathrm{~mm}$; y los promedios en los machos de $31.59 \mathrm{~mm}$ para Shutia y de $27.54 \mathrm{~mm}$ en Balsamar (gráfico 2 y gráfico 3). 
Playa Shutia: Longitud de concha(mm)

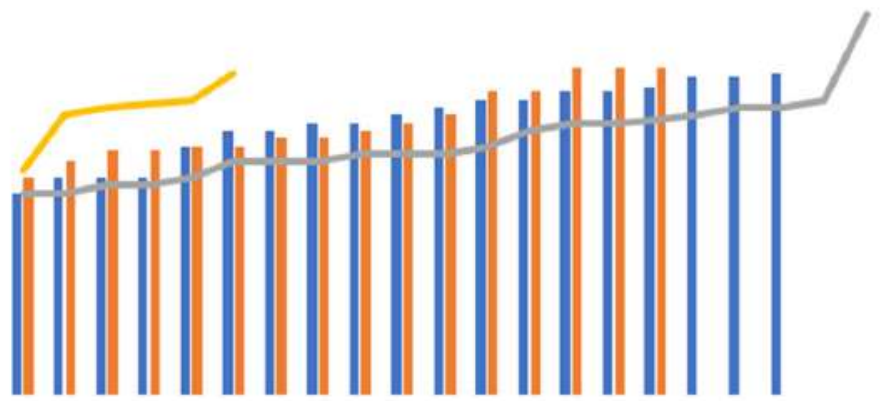

Gráfico 2. Frecuencias longitud de concha machos-hembras.

Fuente: elaboración propia.

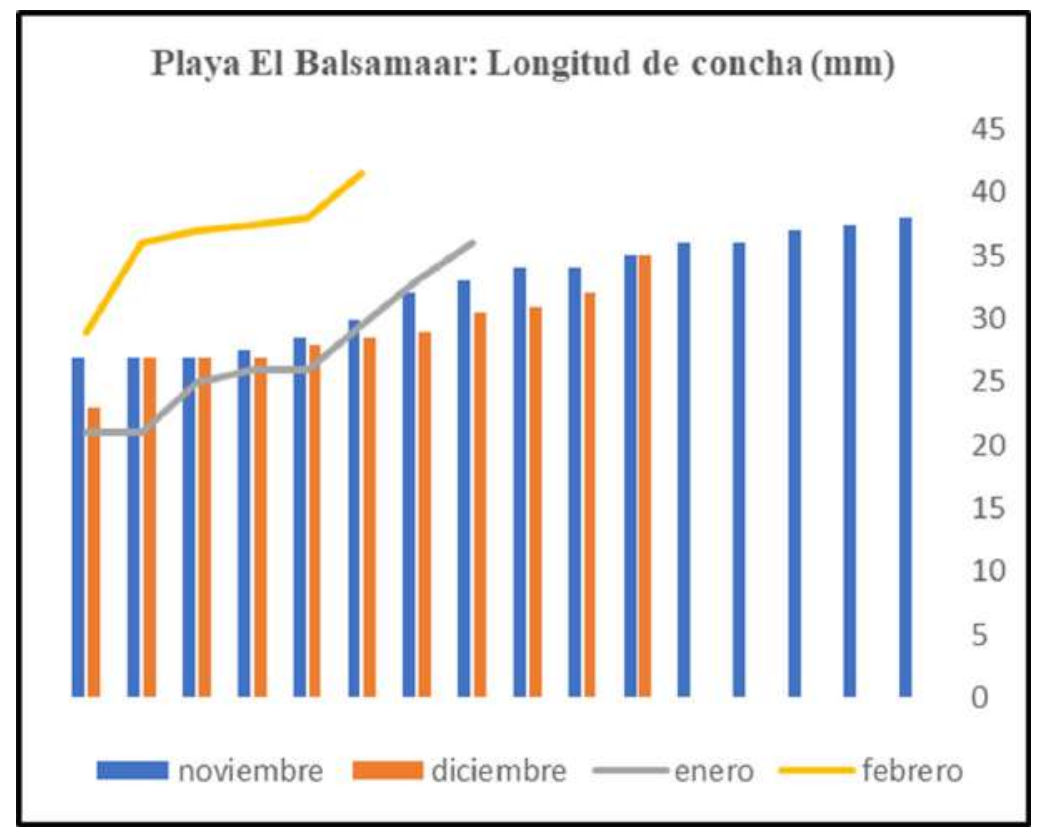

Gráfico 3. Frecuencia longitud de concha macho-hembra.

Fuente: elaboración propia. 
Según Ríos-Jara et al. (1994), en su estudio determinaron que la cantidad de tinte producido se relaciona directamente con el tamaño de la concha; por lo que las hembras poseen mayor cantidad de tinte. Yoshioka (1974; citado en Ríos-Jara, 1994), afirma que las hembras adultas utilizan el tinte para proteger los huevecillos depositados entre las rocas de las playas, por el fuerte olor que expele y el sabor picoso que tiene semejante al ajo que aleja a los depredadores (observación directa en campo); también lo utiliza para adormecer y atrapar a las presas de las que se alimenta.

\section{Discusión}

El método de captura y recaptura ha sido muy utilizado para verificar densidades poblacionales en comunidades biológicas, y muy utilizado en estudios de moluscos. La densidad poblacional es el tamaño del conjunto de organismos respecto a una cierta unidad de espacio, que generalmente se expresa como el número de individuos por unidad de área.

En el estudio realizado en estas dos zonas rocosas se obtuvieron promedios de densidades, 1.36 - 1.02 caracoles $/ \mathrm{m}^{2}$, promedios similares a los obtenidos por Michel-Morfin et al. (2009), con 1.24 y 0.86 caracoles $/ \mathrm{m}^{2}$ en su estudio de bahía Tenacatita (México). Aunque la muestra poblacional en este estudio fue mucho más pequeña que las tomadas por los autores citados.

En cuanto a la abundancia entre machos y hembras, se obtuvo un mayor porcentaje de machos con respecto a hembras, estos resultados son semejantes a los obtenidos por Michel-Morfin et al. (2009), y los realizados en Manzanillo, México (Magaña y Michel-Morfín, 2011). Los porcentajes de la longitud de concha mantienen una relación semejante a los obtenidos por Michel-Morfin et al. (2002). De acuerdo a los resultados estadísticos la población es pobre. Una de las características de ambos sitios es que están influenciados por la presencia humana, factor que puede causar daño directo, ya que el caracol es extraído para uso de carnada para pesca y consumo para alimento (dato obtenido por entrevista hecha a pobladores del lugar).

\section{Conclusión}

Los resultados estadísticos nos arrojan una población pobre en la playa Shutia y playa El Balsamar, lo cual podría deberse que la muestra tomada no fue significativa; esto debido a la dificultad de acceso a las zonas de trabajo. Además es necesario hacer más estudios poblacionales de la especie.

\section{Referencias bibliográficas}

Acevedo, J. y Hernández, C.E. (1988) Aspectos poblacionales y etnobiológicos del caracol Purpura pansa (Gould 1853) en la costa de Oaxaca (Tesis Doctoral). Universidad Nacional Autónoma de México: México. 
Barbero, M.P. (2018) Muestreo de captura-recaptura: diseño, estimación y análisis de librerías en $R$ (Tesis de Licenciatura). Universidad de Sevilla: España.

Garza, R.F., Rodríguez, P.F., Ibáñez, S.G. y Valdés, A. (2007) Demografía del caracol Plicopurpura pansa (Neotaenioglossa: Muricidae) y constitución de la comunidad malacológica asociada en Guerrero, México. Revista Biología Tropical, 55(3-4), 867-878. Disponible en: https://www.scielo. sa.cr/scielo.php?script=sci arttext\&pid=S0034-77442007000300012

Keen, A.M. (1971) Sea shells of tropical West America marine mollusks from Baja California to Perú. California: Editorial Stanford University Press.

Magaña, J. y Michel-Morfin, J.E. (2011) Relocation and monitoring of the purple dye snail Plicopurpura pansa (Gould 1853), in the Tepalcates Channel breakwaters, Manzanillo, Colima, México. Libro de resúmenes de Mollusca 2014 "El encuentro de las Américas", Baja California, México.

Michel-Morfín, J.E., Chávez, A. y González, L. (2002) Estructura de la población, esfuerzo y rendimiento del tinte del caracol Plicopurpura pansa (Gould, 1853) en el Pacífico mexicano. Ciencias marinas, 28, 357-368. Disponible en: https://www.redalyc.org/pdf/480/48028404.pdf

Michel-Morfin, J.E., Hernández, M., Landa-Jaime, V., Arciniega, J., Kosonoy, D. y Flores, R. (2009) Estimation of the abundance and population structure of the purple snail Plicopurpura pansa (Gould, 1853) Comparing two methods. The open marine biology journal, 3(1), 49-58. Disponible en: https:// benthamopen.com/ABSTRACT/TOMBJ-3-49

Payne, E. (2017) La explotación del tinte de caracol y la lucha de los indígenas por su preservación en el Pacífico de Costa Rica (siglos XVI al XIX). Memorias: revista digital de Arqueología e Historia desde el Caribe, (septiembre - diciembre), 142-167. Disponible en: http://www.scielo.org.co/pdf/memor/ n33/1794-8886-memor-33-00142.pdf

Quintanilla, I. (2012) Los Borucas y el teñido de algodón con caracoles marinos. Disponible en: https:// ifigeniaquintanilla.com/2012/05/23/los-boruca-y-el-tenido-de-algodon-con-caracoles-marinos/

Ramírez-Rodríguez, M. y Naegel, L. (2003) Crecimiento del caracol de tinte Plicopurpura pansa en Baja California Sur, México. Ciencias marinas, 29, 283-290. Disponible en: http://www.scielo.org.mx/ scielo.php?script $=$ sci arttext\&pid $=$ S0185-38802003000300003

Ríos-Jara, E.,Álvarez,L., Lizárraga, L.y Michel-Morfín,J.E. (1994) Producción y tiempo de recuperación del tinte de Plicopurpura patula pansa (Neogastropoda: Muricidae) en Jalisco, México. Guadalajara: Universidad de Guadalajara. 
Turok, M., Sigler, A.M., Hernández, C.E., Acevedo, J. y Lara, R. (1988) El caracol púrpura: una tradición milenaria en Oaxaca. México: Dirección General de Culturas Populares. 